\title{
First-in-Human, First-in-Class Phase I Trial of the Anti-CD47 Antibody Hu5F9-G4 in Patients With Advanced Cancers
}

Branimir I. Sikic, MD; Nehal Lakhani, MD, PhD²; Amita Patnaik, MD³; Sumit A. Shah, MD ${ }^{1}$; Sreenivasa R. Chandana, MD, PhD²;

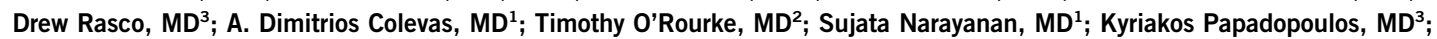
George A. Fisher, MD, PhD ${ }^{1}$; Victor Villalobos, MD, PhD; Susan S. Prohaska, PhD ${ }^{1}$; Maureen Howard, PhD ${ }^{1}$; Muralidhar Beeram, MD³; Mark P. Chao, MD, PhD; ; Balaji Agoram, PhD ; James Y. Chen, MD, PhD ${ }^{5}$; Jie Huang, ScD ${ }^{5}$; Matthew Axt ${ }^{5}$ Jie Liu, PhD, MD; Jens-Peter Volkmer, MD ${ }^{5}$ Ravindra Majeti, MD, PhD ${ }^{1,5}$; Irving L. Weissman, MD ${ }^{1}$; Chris H. Takimoto, MD, PhD ${ }^{5}$; Dana Supan, RN ${ }^{1}$; Heather A. Wakelee, MD ${ }^{1}$; Rhonda Aoki ${ }^{1}$; Mark D. Pegram, MD ${ }^{1}$; and Sukhmani K. Padda, MD ${ }^{1}$

PURPOSE To evaluate the safety, pharmacokinetics, and pharmacodynamics of Hu5F9-G4 (5F9), a humanized IgG4 antibody that targets CD47 to enable phagocytosis.

PATIENTS AND METHODS Adult patients with solid tumors were treated in four cohorts: part A, to determine a priming dose; part B, to determine a weekly maintenance dose; part C, to study a loading dose in week 2; and a tumor biopsy cohort.

RESULTS Sixty-two patients were treated: 11 in part A, 14 in B, 22 in C, and 15 in the biopsy cohort. Part A used doses that ranged from 0.1 to $3 \mathrm{mg} / \mathrm{kg}$. On the basis of tolerability and receptor occupancy studies that showed $100 \%$ CD47 saturation on RBCs, $1 \mathrm{mg} / \mathrm{kg}$ was selected as the priming dose. In subsequent groups, patients were treated with maintenance doses that ranged from 3 to $45 \mathrm{mg} / \mathrm{kg}$, and most toxicities were mild to moderate. These included transient anemia (57\% of patients), hemagglutination on peripheral blood smear (36\%), fatigue $(64 \%)$, headaches (50\%), fever (45\%), chills $(45 \%)$, hyperbilirubinemia (34\%), lymphopenia (34\%), infusionrelated reactions (34\%), and arthralgias (18\%). No maximum tolerated dose was reached with maintenance doses up to $45 \mathrm{mg} / \mathrm{kg}$. At doses of $10 \mathrm{mg} / \mathrm{kg}$ or more, the CD47 antigen sink was saturated by 5F9, and a $5 \mathrm{F9}$ half-life of approximately 13 days was observed. Strong antibody staining of tumor tissue was observed in a patient at $30 \mathrm{mg} / \mathrm{kg}$. Two patients with ovarian/fallopian tube cancers had partial remissions for 5.2 and 9.2 months.

ASSOCIATED

CONTENT

Appendix

Data Supplements

See accompanying article on page 1012

Author affiliations

and support

information (if

applicable) appear

at the end of this

article.

Accepted on January

30, 2019 and

published at jco.org

on February 27, 2019 ;

DOI https://doi.org/10.

1200/JC0.18.02018

Processed as a rapid communication

manuscript.

Clinical trial

information:

NCT02216409.

CONCLUSION $5 F 9$ is well tolerated using a priming dose at $1 \mathrm{mg} / \mathrm{kg}$ on day 1 followed by maintenance doses of up to $45 \mathrm{mg} / \mathrm{kg}$ weekly.

J Clin Oncol 37:946-953. @ 2019 by American Society of Clinical Oncology

\section{INTRODUCTION}

CD47 was first identified as an integrin-associated transmembrane protein that is ubiquitously found in normal and malignant tissues. ${ }^{1}$ Most cancer cells overexpress CD47, and the degree of expression independently correlates with poor clinical outcome in a variety of hematologic and solid tumor malignancies. ${ }^{2,3}$ The binding of CD47 to its receptor signal receptor protein- $\alpha$ (SIRP $\alpha$ ) on macrophages and dendritic cells results in an inhibition of phagocytosis. Thus, CD47 provides a potent "do not eat me" signal that allows for tumor cell evasion of immune destruction by firstresponder phagocytic cells and functions as a dominant macrophage checkpoint. ${ }^{3-5}$ Blockade of CD47$\mathrm{SIRP} \alpha$ signaling in isolation is insufficient to trigger macrophage phagocytosis. Instead, additional prophagocytic signals are required, such as calreticulin and phosphatidylserine, which are frequently found on cancer cells. ${ }^{6,7}$ CD47 also is widely expressed on normal tissues, but because normal cells lack prophagocytic signals, they are not susceptible to CD47mediated phagocytosis. A notable exception is aging RBCs. ${ }^{8}$ Agents that inhibit CD47-SIRP $\alpha$ signaling can induce macrophage phagocytosis of cancer cells both in vitro and in vivo, which results in growth inhibition and regression of a broad range of human cancer xenografts. ${ }^{3,4}$ Therefore, the targeting of CD47 is a novel immunotherapeutic strategy for treating human cancers.

Hu5F9-G4 (5F9) is a humanized IgG4 monoclonal antibody with high affinity for human CD47. ${ }^{9}$ FFmediated blockade of CD47 enhances the phagocytosis of cancer cells by macrophages. In preclinical in vivo models, 5F9 was active against a wide range of 
solid tumors, including cancers of the breast, ovary, colon, liver, brain, and other organs. ${ }^{3-5}$ Potent antitumor activity also was observed in hematologic malignancies, including acute myeloid leukemia (AML), non-Hodgkin lymphoma, cutaneous T-cell lymphoma, acute lymphoblastic leukemia, and multiple myeloma. ${ }^{9}$ In human tumor xenograft models, 5F9 inhibited tumor cell growth and induced remission in established tumors. ${ }^{9}$ In preclinical toxicology studies, the major dose-limiting toxicity (DLT) was an ontarget anemia ${ }^{10}$ that was mitigated by using a priming and maintenance dose schedule. Using this approach, nonhuman primates tolerated $5 \mathrm{F9}$ doses up to $300 \mathrm{mg} / \mathrm{kg}$ without reaching a maximum tolerated dose (MTD). ${ }^{9}$

This report describes the first-in-human phase I trial of 5F9 in patients with advanced solid tumors and lymphomas. The trial consisted of three distinct dose escalation parts. Part A used weekly dosing to determine a tolerable day 1 priming dose. Part B administered the 5F9 priming dose identified in part $A$ followed by escalation of weekly maintenance doses to establish an MTD. At the completion of part B, a tumor biopsy expansion cohort was opened. In part $C$, a loading dose was given on day 11 in addition to weekly 5F9 therapy to enable more-rapid attainment of therapeutic concentrations. The safety, tolerability, and early efficacy results along with summary pharmacokinetics (PK) and pharmacodynamics (PD) data are described here. Detailed PK and PD findings will be reported elsewhere.

\section{PATIENTS AND METHODS}

\section{Patient Selection and Oversight}

Eligible patients were adults 18 years of age or older with histologically or cytologically confirmed advanced solid malignancy or lymphoma previously treated with at least one regimen of systemic therapy, or who refused other systemic therapy, with an Eastern Cooperative Oncology Group performance status of 0 to 2 and for whom no curative therapy was available. Additional eligibility criteria are outlined in the Data Supplement.

This study was approved by the human investigations committee at each institution, and in accordance with assurances filed with and approved by the Department of Health and Human Services. These data are anonymized to protect the identities of the research participants. Informed written consent was obtained from each participant. Patients were enrolled from August 2014 through January 2018.

\section{Study Design and Drug Administration}

The primary objectives were to assess the safety and tolerability of $5 \mathrm{F9}$ and to determine the recommended doses and schedules for additional trials. The secondary objectives were to evaluate the PK and PD profiles and to document antitumor activity. Treatment cycles were 28 days long and continued until disease progression. In part A, the 5F9 starting dose of $0.1 \mathrm{mg} / \mathrm{kg}$ was infused intravenously weekly, with dose escalation conducted in single-patient cohorts for doses less than $3 \mathrm{mg} / \mathrm{kg}$ in a modified accelerated titration design. ${ }^{11}$ The duration of the infusion was 1 hour for doses from 0.1 to $1 \mathrm{mg} / \mathrm{kg}$ and 2 hours for $3 \mathrm{mg} / \mathrm{kg}$. In part B and beyond, all patients received a $1 \mathrm{mg} / \mathrm{kg}$ priming dose on day 1 for 3 hours followed by escalating maintenance doses for 2 hours on day 8 that began at the $3 \mathrm{mg} / \mathrm{kg}$ weekly dose level. After a safe dose was determined in part $B$, a solid tumor expansion cohort of 15 patients was opened, which required mandatory pretreatment and during treatment tumor biopsies.

Beginning in part $B$, routine premedication with acetaminophen and an antihistamine was administered before the first two doses of 5F9. In part C, patients received a loading dose on day 11 of cycle 1 (cohorts of 20,30, and $45 \mathrm{mg} / \mathrm{kg}$ ) in addition to weekly maintenance dosing at the same level. After cycle 1 , if patients had treatment delays longer than 4 weeks, repriming was required.

In part $\mathrm{B}$, dose escalation was performed using a traditional $3+3$ phase I study design. ${ }^{12}$ DLT was defined as any drugrelated adverse event (AE) of grade 3 or greater that occurred from the time of first dose in part $A$, or from the first maintenance doses in parts $B$ and $C$, until the completion of 28 days of therapy (cycle 1 ), with exceptions that are listed in the Appendix. If a DLT was observed, the number of patients at that dose level was expanded from three up to six. Grade 3 anemia was excluded as a DLT because it was expected and anticipated to be transient and reversible. The MTD was defined as the highest dose level explored that induced DLTs in less than 33\% of patients. Up to 10 more patients could be entered to any safe dose level to collect additional safety and PK data.

\section{Study Assessments}

All patients who received at least one 5F9 treatment were evaluated for safety. Standard safety assessments were conducted at baseline, before each weekly infusion, and out to 28 days after the last dose of study drug. Because of the possibility of hemagglutination, peripheral blood smear, visual acuity, and retinal photographic examinations were performed three times weekly for the first two weeks in part A. In part $\mathrm{B}$, peripheral blood smears were examined on days $1,2,8,9,15,22$, and 29, and visual acuity was measured on days 1 and 8 . However, there were no adverse findings on retinal photography as assessed by an ophthalmologist in part $A$. Therefore, retinal photography was not performed in parts $B$ and $C$. Routine laboratory assessments are detailed in the Appendix. AEs were graded according to the National Cancer Institute Common Terminology Criteria for Adverse Events (version 4.03). Tumor measurements were obtained at baseline and every 8 weeks until disease progression or study withdrawal. Response was determined using Response Evaluation Criteria in Solid Tumors (RECIST) version $1.1^{13}$ or the International Working Group response criteria for 
malignant lymphomas. ${ }^{14}$ Details on PK and antidrug antibody (ADA) monitoring as well as PD receptor occupancy studies and tumor biopsy analyses are provided in the Data Supplement.

\section{Statistical Analysis}

This was an open-label, nonrandomized, first-in-human dose escalation phase I trial. All patients exposed to 5F9 were included in the safety analysis, and all patients who had at least one postbaseline tumor assessment were considered evaluable for tumor response assessment.

\section{RESULTS}

\section{Patient Characteristics}

The characteristics of all 62 treated patients are listed in Table 1. Female patients comprised $65 \%$ of participants

TABLE 1. Patient Demographics and Baseline Characteristics

\begin{tabular}{|c|c|c|}
\hline Characteristic & No. & $\%$ \\
\hline No. of patients treated & 62 & 100 \\
\hline Median age, years (range) & $60(35-84)$ & \\
\hline \multicolumn{3}{|l|}{ Sex } \\
\hline Male & 22 & 35 \\
\hline Female & 40 & 65 \\
\hline \multicolumn{3}{|l|}{ Ethnicity } \\
\hline Hispanic or Latino & 4 & 6.5 \\
\hline Not Hispanic or Latino & 58 & 93.5 \\
\hline \multicolumn{3}{|l|}{ Race } \\
\hline White & 49 & 79.0 \\
\hline Asian & 11 & 17.7 \\
\hline Black & 2 & 3.2 \\
\hline \multicolumn{3}{|l|}{ ECOG performance status } \\
\hline 0 & 19 & 30 \\
\hline 1 & 42 & 68 \\
\hline 2 & 1 & 2 \\
\hline Median No. of prior systemic treatments (range) & $5(1-18)$ & \\
\hline \multicolumn{3}{|l|}{ Tumor type } \\
\hline Colorectal & 18 & 29.0 \\
\hline Ovarian & 13 & 21.0 \\
\hline Salivary & 5 & 8.1 \\
\hline Breast & 5 & 8.1 \\
\hline Head and neck & 4 & 6.5 \\
\hline Pancreatic & 4 & 6.5 \\
\hline Lung & 2 & 3.2 \\
\hline Skin & 2 & 3.2 \\
\hline DLBCL & 2 & 3.2 \\
\hline Other & 7 & 11.3 \\
\hline
\end{tabular}

Abbreviations: DLBCL, diffuse large B-cell lymphoma; ECOG, Eastern Cooperative Oncology Group. and included 13 with ovarian cancer and five with breast cancer. The most frequent tumor type was colorectal cancer. Nine patients had salivary gland or head and neck cancers, and two had diffuse large B-cell lymphoma (DLBCL). Patients were heavily pretreated, having received a median of five systemic treatments (range, one to 18 treatments) before study enrollment. The Eastern Cooperative Oncology Group performance status was 0 in 19 patients (30\%), 1 in 42 patients (68\%), and 2 in one patient (2\%).

\section{Safety}

The treatment cohorts and DLTs are listed in Table 2. Two DLTs were observed in part A at $3 \mathrm{mg} / \mathrm{kg}$, including one patient who developed grade 3 right-side upper-abdominal pain during the first infusion of $5 \mathrm{F9}$ and one patient with grade 3 hemagglutination defined as grade 2 headache associated with hemagglutination on the peripheral blood smear. However, no DLTs were observed in the six patients treated at $1 \mathrm{mg} / \mathrm{kg}$, so this dose was selected as the day 1 priming dose for use in all subsequent parts of the study.

In part B, weekly maintenance doses were administered at 3 , 10 , and $20 \mathrm{mg} / \mathrm{kg}$ beginning on day 8 after the priming dose. One patient at $3 \mathrm{mg} / \mathrm{kg}$ had transient grade 3 elevated bilirubin without changes in serum transaminases, which at the time was defined as a DLT. A second patient treated at $20 \mathrm{mg} / \mathrm{kg}$ had grade 3 hemagglutination (grade 2 headache with hemagglutination on peripheral blood smear), but no other DLTs occurred in the six patients treated at this dose level. Fifteen additional patients were treated with $20 \mathrm{mg} / \mathrm{kg}$ in the tumor biopsy expansion cohort to collect additional PD data, and no DLTs were observed. In part C, one patient at $20 \mathrm{mg} / \mathrm{kg}$ experienced grade 3 elevated bilirubin, but no additional DLTs occurred despite escalation to $30 \mathrm{mg} / \mathrm{kg}$ and $45 \mathrm{mg} / \mathrm{kg}$ in nine and six patients, respectively. Thus, no MTD was reached in parts $\mathrm{B}$ or $\mathrm{C}$. On the basis of this favorable safety profile and the accumulated PK data that demonstrated steady-state dose levels well above the targeted threshold of $200 \mu \mathrm{g} / \mathrm{mL}^{15}$ (Fig 1A), a maintenance dose of $30 \mathrm{mg} / \mathrm{kg}$ was recommended for additional clinical evaluation.

The combined AE profile from 44 patients treated with 5F9 maintenance doses of 20,30, and $45 \mathrm{mg} / \mathrm{kg}$ are listed in Table 3. Overall, the most common 5F9 toxicity was the expected on-target anemia, which occurred in 57\% of patients treated with doses that ranged from 20 to $45 \mathrm{mg} / \mathrm{kg}$ (13\% of patients with grade 3 ). Typically, a modest, predictable fall in hemoglobin ( 1 to $2 \mathrm{~g} / \mathrm{dL}$ ) occurred after the $1 \mathrm{mg} / \mathrm{kg}$ priming dose followed by a rapid compensatory reticulocytosis, as illustrated in a single patient in Figure 1B. 5 F9 treatment was not associated with thrombocytopenia. In the 52 patients treated for at least 4 weeks, the median percent change in platelet count from baseline on day 29 at the end of the first cycle was $-3.2 \%$ (range, $-54.3 \%$ to $91.2 \%$ ). Five patients received concomitant anticoagulant therapy, but no associated bleeding or thrombocytopenia was observed. 
TABLE 2. Treatment Cohorts and DLTS

\begin{tabular}{|c|c|c|c|}
\hline Part and Dose Cohort & $\begin{array}{l}\text { Patients, } \\
\text { No. }\end{array}$ & $\begin{array}{l}\text { Infusions, } \\
\text { No. }\end{array}$ & $\begin{array}{c}\text { Patients } \\
\text { With } \\
\text { DLTs, No. }\end{array}$ \\
\hline \multicolumn{4}{|c|}{ Part A: weekly dose (prime), mg/kg } \\
\hline 0.1 & 1 & 72 & 0 \\
\hline 0.3 & 2 & 11 & 0 \\
\hline 1 & 6 & 70 & 0 \\
\hline 3 & 2 & 2 & $2^{*} \dagger$ \\
\hline \multicolumn{4}{|c|}{$\begin{array}{l}\text { Part B: prime, maintenance } \\
\text { doses, mg/kg }\end{array}$} \\
\hline 1,3 & 4 & 20 & $1 \ddagger$ \\
\hline 1,10 & 3 & 112 & 0 \\
\hline 1,20 & 7 & 78 & $1 \dagger$ \\
\hline \multicolumn{4}{|c|}{$\begin{array}{l}\text { Tumor biopsy cohort: prime, } \\
\text { maintenance doses, mg/kg }\end{array}$} \\
\hline 1,20 & 15 & 197 & 0 \\
\hline \multicolumn{4}{|c|}{$\begin{array}{l}\text { Part C: prime, loading, maintenance } \\
\quad \text { doses, } \mathrm{mg} / \mathrm{kg}\end{array}$} \\
\hline $1,20,20$ & 7 & 130 & $1 \ddagger$ \\
\hline $1,30,30$ & 9 & 129 & 0 \\
\hline $1,45,45$ & 6 & 54 & 0 \\
\hline Total & 62 & 875 & 5 \\
\hline
\end{tabular}

Abbreviation: DLT, dose-limiting toxicity.

*Grade 3 pain, right-side upper abdomen.

tGrade 3 hemagglutination (grade 2 headache with hemagglutination on peripheral blood smear).

$\ddagger$ Grade 3 elevated bilirubin (unconjugated)/blood bilirubin increase.

Hemagglutination on the peripheral blood smear was noted in $36 \%$ of patients but was not associated with significant clinical toxicities ( $2 \%$ grade 3 ). Specifically, no neurologic, renal, or cutaneous abnormalities were noted.

Other common toxicities were grade 1 and 2 fatigue (64\%), headaches $(50 \% ; 2 \%$ grade 3$)$, fever $(45 \%)$, chills $(45 \%)$, hyperbilirubinemia (34\%), and arthralgias (18\%). Unconjugated hyperbilirubinemia that was not associated with other liver function test abnormalities was common after the 5F9 priming dose; however, it generally returned to baseline within a few days. Transient elevation in unconjugated bilirubin was likely due to the expected phagocytosis of aged RBCs that result from blockade of $\mathrm{CD} 47$. As such, in part $\mathrm{B}$, the protocol was amended to exclude grade 3 hyperbilirubinemia from the DLT definition. First- or second-dose infusion-related reactions (IRRs) occurred in $34 \%$ of patients, predominantly in those enrolled in the $20 \mathrm{mg} / \mathrm{kg}$ and higher dose cohorts. These were characterized by symptoms such as chills, fever/pyrexia, photopsia, back pain, and headaches that generally lasted a day or two after the associated infusion and were managed with symptomatic supportive care. Overall, only one patient underwent dose reduction from 30 to $20 \mathrm{mg} / \mathrm{kg}$ in part $C$ because of an IRR, and this patient was able to receive further treatment without problems. One additional patient withdrew consent because of infusion-related AEs. However, all other patients who experienced IRRs were able to continue treatment at their assigned dose level.

Transient grade 3 IRRs that responded to symptomatic treatment and that did not require dose reductions were not considered DLTs. No risk factors for IRRs were identified. After the first three patients were treated in the part $C$ loading dose cohort at $30 \mathrm{mg} / \mathrm{kg}$, routine antihistamine and acetaminophen premedication was mandated for all new patients before the initial priming dose of $1 \mathrm{mg} / \mathrm{kg}$ on day 1 and before the second higher dose on day 8 . However, even with routine prophylaxis, grade 1 or 2 IRRs that were managed symptomatically were common and reported in seven of 12 premedicated patients treated in part $C$ at 30 or $45 \mathrm{mg} / \mathrm{kg}$. Lymphopenia was found in 36\% of patients without apparent clinical consequences. No treatmentrelated deaths occurred on study.

Hemagglutination was seen on peripheral blood smear typically after the first or second 5F9 dose. However, there were no clear clinical sequelae. Specifically, no treatmentrelated peripheral vascular symptoms, changes in renal function, deep venous thromboses, pulmonary emboli, or other thrombotic events were observed. Furthermore, serial retinal photographs were collected over seven time points in the first cycle to assess for microvascular changes, but no such findings were noted, so the protocol was amended to remove retinal photography evaluation in part $\mathrm{B}$.

\section{PK and ADA Analysis}

Similar to the PK in nonhuman primates, 5F9 exhibited nonlinear PK behavior as a result of the CD47 antigen sink, which resulted in a short apparent half-life at doses of 0.1 to $3 \mathrm{mg} / \mathrm{kg}$ of $5 \mathrm{Fg}$ (Fig 1A; and online only Appendix Table A1). However, doses of $10 \mathrm{mg} / \mathrm{kg}$ and higher saturated this sink, which extended the 5F9 half-life to approximately 13 days, consistent with other humanized monoclonal antibodies. ${ }^{16}$ At these doses, free antibody levels exceeded the antitumor activity threshold of $200 \mu \mathrm{g} / \mathrm{mL}$ identified from PK modeling., ${ }^{9,15}, 19$ Serum samples from six (9.7\%) of 62 patients tested positive for ADA, but there was no impact on 5F9 PK and no apparent clinical symptoms. ${ }^{15}$ Detailed PK profiling will be presented elsewhere.

\section{PD and Tissue Biopsy Analysis}

Studies of RBC occupancy showed complete saturation of CD47 on RBCs at doses of $1 \mathrm{mg} / \mathrm{kg}$ and higher (Fig 2A), which also supported $1 \mathrm{mg} / \mathrm{kg}$ as the initial 5F9 priming dose. In contrast, maximal saturation of CD47 on WBCs was only observed after higher maintenance doses (Fig 2B). Doses of $30 \mathrm{mg} / \mathrm{kg}$ maximally saturated CD47 on circulating WBCs.

In addition, anti-lgG4 staining demonstrated strong antibody penetration into metastatic tumor tissue in an axillary 


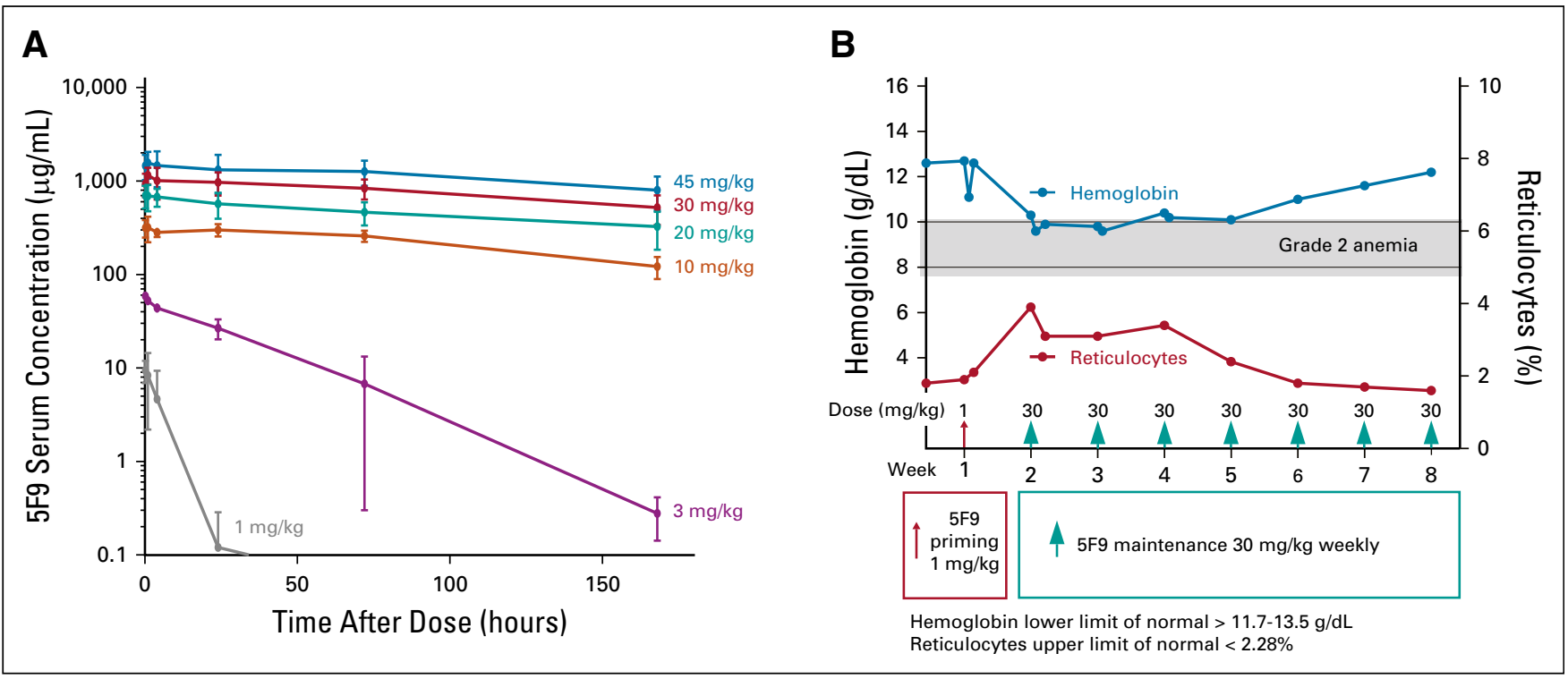

FIG 1. Hu5F9-G4 (5F9) pharmacokinetics and effects on hemoglobin and reticulocyte levels. (A) Concentration of 5F9 (mean \pm standard deviation) versus time during week 5 over the evaluated weekly dose range (1 to $45 \mathrm{mg} / \mathrm{kg}$; number of patients per dose range, three to 30 ). (B) Anemia with compensatory reticulocytosis in one patient.

lymph node from a patient with metastatic ovarian cancer (Fig 2C). This patient was treated with $30 \mathrm{mg} / \mathrm{kg} 5 \mathrm{F9}$, and the biopsy sample was obtained after 5 weeks of treatment. These collected data support the recommended dose of 30 $\mathrm{mg} / \mathrm{kg}$ for phase II evaluation.

\section{Antitumor Activity}

Two patients with clear cell ovarian and fallopian tube carcinomas had confirmed partial responses by RECIST version 1.1 (Figs $3 \mathrm{~A}$ and $3 \mathrm{~B}$ ), with time to progression of 5.2 months and 9.2 months, respectively. These patients had reductions in target lesions of $50 \%$ and $44 \%$. Their cancer antigen 125 tumor markers dropped from 338 to 70 $\mathrm{U} / \mathrm{mL}$ and 890 to $103 \mathrm{U} / \mathrm{mL}$, respectively. Both patients were heavily pretreated with more than six prior lines of systemic therapy, and both received $5 \mathrm{F9}$ at $20 \mathrm{mg} / \mathrm{kg}$. A third patient with DLBCL at the $20 \mathrm{mg} / \mathrm{kg}$ maintenance dose had a mixed response by International Working Group

TABLE 3. Adverse Event Summary for Patients Treated With Maintenances Doses of $20 \mathrm{mg} / \mathrm{kg}$ or Higher

Patients Treated, No. (\%)

\section{Part B, Biopsy Cohort, and Part C \\ Prime $+20 \mathrm{mg} / \mathrm{kg}$ Maintenance Dose ( $n=29)$}

\begin{tabular}{|c|c|c|c|c|c|c|c|c|c|}
\hline \multirow[b]{2}{*}{ Adverse Event* } & & & \\
\hline & All Grades & Grade 3 & Grade 4 & All Grades & Grade 3 & Grade 4 & All Grades & Grade 3 & Grade 4 \\
\hline Hemagglutination & $12(41)$ & $1(3)$ & 0 & $2(22)$ & 0 & 0 & $2(33)$ & 0 & 0 \\
\hline Thrombocytopenia & $5(17)$ & 0 & 0 & 0 & 0 & 0 & 0 & 0 & 0 \\
\hline Lymphocyte count decreased & $4(14)$ & $4(14)$ & 0 & $3(33)$ & $2(22)$ & $1(11)$ & $1(17)$ & $1(17)$ & 0 \\
\hline Headache & $11(38)$ & 0 & 0 & $6(67)$ & $1(11)$ & 0 & $4(67)$ & 0 & 0 \\
\hline Nausea & $3(10)$ & 0 & 0 & $2(22)$ & 0 & 0 & $3(50)$ & 0 & 0 \\
\hline Fatigue & $18(62)$ & 0 & 0 & $6(67)$ & 0 & 0 & $4(67)$ & 0 & 0 \\
\hline Fever & $14(48)$ & 0 & 0 & $4(44)$ & 0 & 0 & $2(33)$ & 0 & 0 \\
\hline
\end{tabular}

${ }^{*}$ Adverse events occurred in $>15 \%$ of patients across all three cohorts listed $(n=44)$ and selected adverse events of interest. 


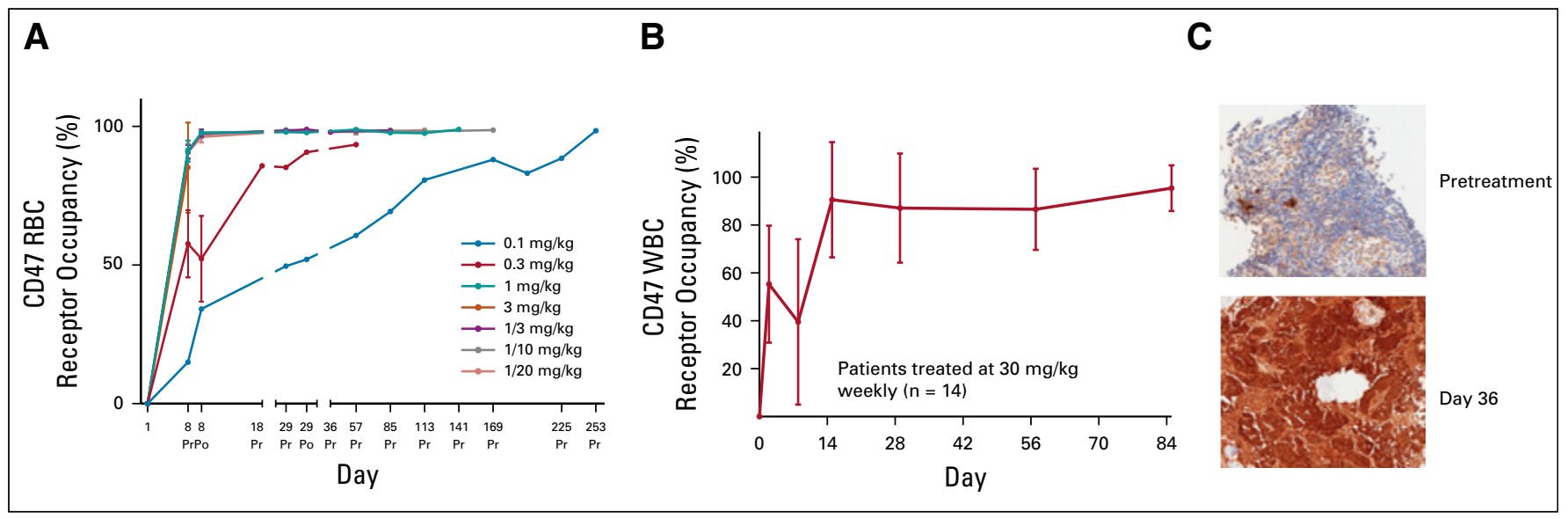

FIG 2. Pharmacodynamics of Hu5F9-G4. (A) RBC receptor occupancy. (B) WBC receptor occupancy. (C) lgG4 immunohistochemical staining in a lymph node with tumor involvement obtained from a 70-year-old patient with ovarian cancer treated with $30 \mathrm{mg} / \mathrm{kg}$ of $5 \mathrm{F9}$. She remained on study for 7 months with a best overall response of stable disease. $1 / 3$ is $1 \mathrm{mg} / \mathrm{kg}$ priming dose and $3 \mathrm{mg} / \mathrm{kg}$ weekly dosing. 1/10 is $1 \mathrm{mg} / \mathrm{kg}$ priming dose and $10 \mathrm{mg} / \mathrm{kg}$ weekly dosing. $1 / 20$ is $1 \mathrm{mg} / \mathrm{kg}$ priming dose and $20 \mathrm{mg} / \mathrm{kg}$ weekly dosing. Po, post-infusion sample collection; Pr, pre-infusion sample collection.

criteria. This heavily pretreated patient with non-Hodgkin lymphoma had bulky lesions in the chest that decreased in size but had a solitary inguinal lymph node that increased while on treatment.

\section{DISCUSSION}

The anti-CD47 antibody 5F9 is well-tolerated in patients with solid tumors and lymphoma when administered using a priming and maintenance dose regimen. An MTD was not defined, even at doses up to $45 \mathrm{mg} / \mathrm{kg}$ weekly. The recommended 5F9 priming dose of $1 \mathrm{mg} / \mathrm{kg}$ on day 1 achieved maximal CD47 receptor occupancy on RBCs. Receptor occupancy on circulating WBCs was not available at low maintenance doses, but available data confirmed complete WBC receptor occupancy at weekly maintenance dosing of $30 \mathrm{mg} / \mathrm{kg}$ beginning on day 8 . A tissue biopsy sample of a lymph node with metastatic ovarian cancer in one patient demonstrated excellent penetration of 5F9 into the tumor microenvironment. At doses above $10 \mathrm{mg} / \mathrm{kg}$, the PK of $5 F 9$ was linear, and increases in drug exposure were dose proportional. The resulting long terminal half-life of approximately
13 days supports every 2-week dosing after saturating concentrations are achieved.

The most common toxicity was expected, on-target, mild, transient, and predictable anemia, which was mitigated by administering priming and maintenance doses in cycle 1. After the initial priming dose, hemoglobin levels dropped on average by approximately $2 \mathrm{~g} / \mathrm{dL}$ presumably because of RBC phagocytosis by macrophages in the liver and spleen. However, this was immediately followed by a rapid compensatory reticulocytosis as younger RBCs were released into circulation. Younger RBCs are unperturbed by CD47 blockade because they lack prophagocytic signals that accumulate on older RBCs. Despite continued 5F9 dosing, hemoglobin levels typically remained stable beyond the first week, and in many patients, the anemia gradually resolved to baseline as the circulating RBC population shifted to a younger average age. Hemagglutination on peripheral blood smear was noted in some patients, typically after the first or second dose, but there was no consistent association with clinical sequelae. Blood transfusions were infrequently required in this mostly solid tumor population, with four of

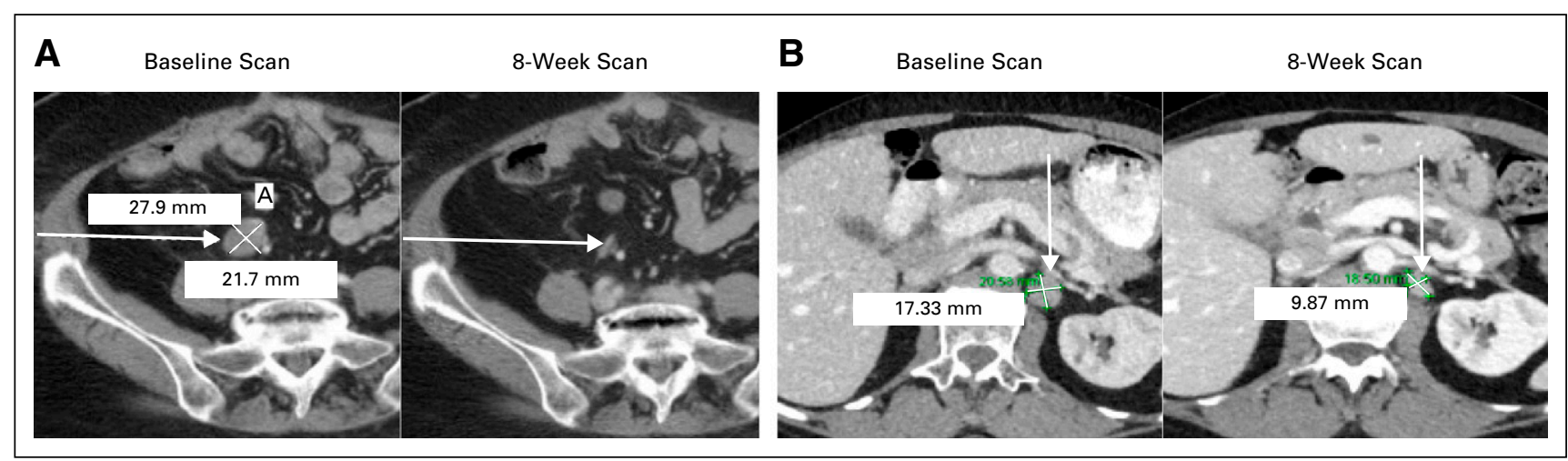

FIG 3. Hu5F9-G4 partial responses observed in (A) a patient with clear cell ovarian cancer and (B) a patient with fallopian tube cancer. 
62 patients receiving RBC transfusions. However, early clinical experience with 5F9 in patients with refractory end-stage $\mathrm{AML}^{17}$ suggests that blood product support can readily be administered to patients who receive 5F9 therapy.

Most 5F9-related AEs were mild to moderate in severity, and $5 F 9$ infusions were easily administered in an outpatient setting. At dose levels of $20 \mathrm{mg} / \mathrm{kg}$ and higher, the commonly observed AEs were headache, fatigue, fever, and chills during the infusions, with most occurring within the first cycle. Pretreatment with acetaminophen and diphenhydramine lowered the incidence and severity of these reactions, and this pretreatment is recommended before the first two doses of 5F9. A few patients experienced abdominal, back, or chest pains during infusion that were not associated with other clinical findings and often was mitigated by temporarily stopping or slowing the rate of infusion. No treatment-related autoimmune phenomena (pneumonitis, colitis, or hepatitis) analogous to those reported with programmed cell death 1/programmed cell death-ligand 1 checkpoint inhibitors were observed. Despite the widespread expression of CD47 on normal tissues, patients tolerated 5F9 well presumably because normal cells, unlike their malignant counterparts, lack prophagocytic signals and thus are not susceptible to CD47 blockade. Of note, no dose-related thrombocytopenia or neutropenia was observed. This favorable safety profile suggests that 5F9 may be readily combined with other antitumor antibodies, which is consistent with emerging clinical reports. ${ }^{18}$

Of note, single-agent 5F9 induced objective tumor responses in two patients, one with clear cell ovarian cancer and one with a fallopian tube cancer. Both patients were

\section{AFFILIATIONS}

${ }^{1}$ Stanford University School of Medicine, Stanford, CA

${ }^{2}$ South Texas Accelerated Therapeutics Midwest, Grand Rapids, MI

${ }^{3}$ South Texas Accelerated Therapeutics, San Antonio, TX

${ }^{4}$ University of Colorado Cancer Center, Aurora, CO

${ }^{5}$ Forty Seven, Menlo Park, CA

\section{CORRESPONDING AUTHOR}

Branimir I. Sikic, MD, Division of Oncology, Department of Medicine, Stanford University School of Medicine, 269 Campus Dr, CCSR-1105c, Stanford, CA 94305; e-mail: brandy@stanford.edu.

\section{PRIOR PRESENTATION}

Presented at the American Society of Clinical Oncology 2018 Annual Meeting, Chicago, IL, June 1-5, 2018.

\section{SUPPORT}

Supported by grants from the National Institutes of Health (Stanford Clinical and Translational Research Unit), California Institute for Regenerative Medicine, and Forty Seven. heavily pretreated with more than six lines of systemic therapy, including multiple lines of platinum-based chemotherapy. However, all phase I first-in-human studies have limitations because of the heterogeneity of patients who may not reflect broader phase II and III study populations because they are selected for more indolent disease, including many who have participated in multiple prior phase I studies. Therefore, further evaluation of 5F9 is ongoing in an additional cohort of patients with ovarian cancer.

Detailed translational studies that analyze treatment-related changes in the tumor microenvironment in biopsy samples from patients in this trial are ongoing. Additional trials are ongoing that are evaluating 5F9 therapy in patients with ovarian cancer, AML, and cutaneous T-cell lymphoma. 5F9 generated objective responses in patients with solid tumors in this phase I trial; however, its optimal therapeutic impact likely will be in combination with other anticancer therapies. ${ }^{18}$ Ongoing combination trials are evaluating 5F9 plus rituximab in B-cell lymphomas (ClinicalTrials.gov identifier: NCT02953509), ${ }^{18}$ with cetuximab in colorectal cancers (ClinicalTrials.gov identifier: NCT02953782), with azacitidine in treatment-naïve $A M L$ and myelodysplastic syndrome (ClinicalTrials.gov identifier: NCT03248479), and with the programmed cell death-ligand 1 checkpoint inhibitor avelumab in patients with ovarian cancer (ClinicalTrials.gov identifier: NCT03558139). 5F9 has received fast-track designation by the US Food and Drug Administration for further development in DLBCL and follicular lymphoma. In summary, enhancement of macrophage phagocytosis of tumor cells by blocking CD47 with 5F9 is a promising new cancer therapeutic strategy.

AUTHORS' DISCLOSURES OF POTENTIAL CONFLICTS OF INTEREST AND DATA AVAILABILITY STATEMENT

Disclosures provided by the authors and data availability statement (if applicable) are available with this article at DOI https://doi.org/10.1200/ JC0.18.02018.

\section{AUTHOR CONTRIBUTIONS}

Conception and design: Branimir I. Sikic, Sumit A. Shah, A. Dimitrios Colevas, Sujata Narayanan, George A. Fisher, Victor Villalobos, Susan S. Prohaska, Maureen Howard, Mark P. Chao, Jie Huang, Jie Liu, Jens-Peter Volkmer, Ravindra Majeti, Irving L. Weissman, Chris H. Takimoto, Mark D. Pegram, Sukhmani K. Padda

Financial support: Branimir I. Sikic, Ravindra Majeti, Chris H. Takimoto Administrative support: Branimir I. Sikic, Nehal Lakhani, Sreenivasa R. Chandana, Ravindra Majeti, Chris H. Takimoto, Rhonda Aoki

Provision of study material or patients: Branimir I. Sikic, Nehal Lakhani, Drew Rasco, A. Dimitrios Colevas, Timothy O'Rourke, Kyriakos Papadopoulos, George A. Fisher, Susan S. Prohaska, Heather A. Wakelee, Mark D. Pegram

Collection and assembly of data: Branimir I. Sikic, Nehal Lakhani, Amita Patnaik, Sumit A. Shah, Sreenivasa R. Chandana, Drew Rasco, A. Dimitrios Colevas, Timothy O'Rourke, Sujata Narayanan, Kyriakos Papadopoulos, George A. Fisher, Victor Villalobos, Maureen Howard, Muralidhar Beeram, Mark P. Chao, Balaji Agoram, James Y. Chen, Jie 
Huang, Matthew Axt, Jens-Peter Volkmer, Chris H. Takimoto, Dana Supan, Heather A. Wakelee, Rhonda Aoki, Mark D. Pegram, Sukhmani K. Padda

Data analysis and interpretation: Branimir I. Sikic, Nehal Lakhani, Amita Patnaik, Sumit A. Shah, A. Dimitrios Colevas, Sujata Narayanan, George A. Fisher, Maureen Howard, Mark P. Chao, Balaji Agoram, James Y. Chen, Jie Huang, Jens-Peter Volkmer, Irving L. Weissman, Chris H. Takimoto, Heather A. Wakelee, Mark D. Pegram, Sukhmani K. Padda Manuscript writing: All authors
Final approval of manuscript: All authors

Accountable for all aspects of the work: All authors

\section{ACKNOWLEDGMENT}

We thank Susan Jerian, MD, and David Essayan, MD, of ONCORD for their advice on study design. We are most grateful to the participating patients and their family members and to all the nurses, investigators, and study staff who contributed to this clinical study.

\section{REFERENCES}

1. Brown EJ, Frazier WA: Integrin-associated protein (CD47) and its ligands. Trends Cell Biol 11:130-135, 2001

2. Chao MP, Alizadeh AA, Tang C, et al: Anti-CD47 antibody synergizes with rituximab to promote phagocytosis and eradicate non-Hodgkin lymphoma. Cell 142: 699-713, 2010

3. Willingham SB, Volkmer JP, Gentles AJ, et al: The CD47-signal regulatory protein alpha (SIRPa) interaction is a therapeutic target for human solid tumors. Proc Natl Acad Sci U S A 109:6662-6667, 2012

4. Chao MP, McKenna KM, Cha A, et al: The anti-CD47 antibody Hu5F9-G4 is a novel immune checkpoint inhibitor with synergistic efficacy in combination with clinically active cancer targeting antibodies. Cancer Immunol Res 4, 2016 (abstr PR13)

5. Majeti R, Chao MP, Alizadeh AA, et al: CD47 is an adverse prognostic factor and therapeutic antibody target on human acute myeloid leukemia stem cells. Cell 138:286-299, 2009

6. Chen Q, Fang X, Jiang C, et al: Thrombospondin promoted anti-tumor of adenovirus-mediated calreticulin in breast cancer: Relationship with anti-CD47. Biomed Pharmacother 73:109-115, 2015

7. Chao MP, Jaiswal S, Weissman-Tsukamoto R, et al: Calreticulin is the dominant pro-phagocytic signal on multiple human cancers and is counterbalanced by CD47. Sci Transl Med 2:63ra94, 2010

8. Oldenborg PA, Zheleznyak A, Fang YF, et al: Role of CD47 as a marker of self on red blood cells. Science 288:2051-2054, 2000

9. Liu J, Wang L, Zhao F, et al: Pre-clinical development of a humanized anti-CD47 antibody with anti-cancer therapeutic potential. PLoS One 10:e0137345, 2015

10. Olsson M, Oldenborg PA: CD47 on experimentally senescent murine RBCs inhibits phagocytosis following Fcgamma receptor-mediated but not scavenger receptor-mediated recognition by macrophages. Blood 112:4259-4267, 2008

11. Simon R, Freidlin B, Rubinstein L, et al: Accelerated titration designs for phase I clinical trials in oncology. J Natl Cancer Inst 89:1138-1147, 1997

12. Le Tourneau C, Lee JJ, Siu LL: Dose escalation methods in phase I cancer clinical trials. J Natl Cancer Inst 101:708-720, 2009

13. Eisenhauer EA, Therasse P, Bogaerts J, et al: New response evaluation criteria in solid tumours: Revised RECIST guideline (version 1.1). Eur J Cancer 45: 228-247, 2009

14. Barrington SF, Mikhaeel NG, Kostakoglu L, et al: Role of imaging in the staging and response assessment of lymphoma: Consensus of the International Conference on Malignant Lymphomas Imaging Working Group. J Clin Oncol 32:3048-3058, 2014

15. Agoram B, Wang B, Sikic BI, et al: Pharmacokinetics of Hu5F9-G4, a first-in-class anti-CD47 antibody, in patients with solid tumors and lymphomas. J Clin Oncol 36, 2018 (suppl; abstr 2525)

16. Wang W, Wang EQ, Balthasar JP: Monoclonal antibody pharmacokinetics and pharmacodynamics. Clin Pharmacol Ther 84:548-558, 2008

17. Vyas $P$, Knapper S, Kelly R, et al: Initial phase 1 results of the first-in-class anti-CD47 antibody Hu5F9-G4 in relapsed/refractory acute myeloid leukemia patients. EHA Learning Center 2018 (abstr PF232)

18. Advani R, Flinn I, Popplewell L, et al: CD47 blockade by Hu5F9-G4 and rituximab in non-Hodgkin's lymphoma. N Engl J Med 379:1711-1721, 2018

19. Buckeridge C, Duvvuri S, Denney WS: Simple, automatic noncompartmental analysis: The PKNCA R package. J Pharmacokinet Pharmacodyn 42:S65, 2015

\section{Cancer.Net Mobile App for Patients}

Cancer.Net's award-winning app is the mobile companion for patients to stay informed about cancer and to organize important personal data often needed for visits to physicians. It includes interactive tools to help patients get answers to important questions, track side effects, and manage medications. Patients using Spanish language - enabled devices can also access the tools and information in Spanish. Direct your patients to cancer.net/app to download the Cancer.Net mobile app. 
The following represents disclosure information provided by authors of this manuscript. All relationships are considered compensated. Relationships are self-held unless noted. I = Immediate Family Member, Inst = My Institution. Relationships may not relate to the subject matter of this manuscript. For more information about ASCO's conflict of interest policy, please refer to www.asco.org/rwc or ascopubs.org/jco/site/ifc.

Branimir I. Sikic

Consulting or Advisory Role: Threshold Pharmaceuticals, Immune Design Research Funding: Forty Seven (Inst), Celldex Therapeutics (Inst), Gilead Sciences (Inst), Basilea Pharmaceutica (Inst), Genentech (Inst), Roche (Inst), Sanofi (Inst)

\section{Nehal Lakhani}

Research Funding: Alexo Therapeutics (Inst), Ascentage Pharma (Inst), Asana BioSciences (Inst), BeiGene (Inst), Constellation Pharmaceuticals (Inst), Alexion Pharmaceuticals (Inst), Cerulean Pharma (Inst), Forty Seven (Inst), Loxo Oncology (Inst), Macrogenics (Inst), Merck (Inst), Pfizer (Inst), Regeneron Pharmaceuticals (Inst), TaiRx (Inst), Apexian Pharmaceuticals (Inst), Formation Biologics (Inst), Coordination Therapeutics (Inst), Symphogen (Inst), CytomX Therapeutics (Inst), InhibRx (Inst), Incyte (Inst), Jounce Therapeutics (Inst), Livzon Pharmaceutical Group (Inst), Northern Biologics (Inst), TESARO (Inst)

\section{Amita Patnaik}

Consulting or Advisory Role: Bayer AG, Novartis, Genentech (I), Roche (I), Merck

Research Funding: Merck (Inst), Pfizer (Inst), Eli Lilly (Inst), Plexxikon (Inst), Corvus Pharmaceuticals (Inst), TESARO (Inst), AbbVie (Inst), Forty Seven (Inst), Five Prime Therapeutics (Inst), Infinity Pharmaceuticals (Inst), Proximagen (Inst), Pieris Pharmaceuticals (Inst), Surface Oncology (Inst), Livzon Pharmaceutical Group (Inst), Vigeo Therapeutics (Inst), Astellas Pharma (Inst), KLUS Pharma (Inst), Symphogen (Inst), Syndax (Inst)

Sumit A. Shah

Stock and Other Ownership Interests: Grand Rounds

Sreenivasa R. Chandana

Consulting or Advisory Role: Ipsen, AstraZeneca, Eli Lilly

Speakers' Bureau: Ipsen

Research Funding: Novocure (Inst)

\section{Drew Rasco}

Employment: START

Consulting or Advisory Role: TaiRx, Boehringer Ingelheim, Eli Lilly

Research Funding: Celgene (Inst), Millennium Pharmaceuticals (Inst), Rexahn Pharmaceuticals (Inst), Santa Maria Biotherapeutics (Inst), Five Prime Therapeutics (Inst), Pharmacyclics (Inst), Asana Biosciences (Inst), Eisai (Inst), Aeglea BioTherapeutics (Inst), Merck (Inst), Ascentage Pharma (Inst), Macrogenics (Inst), Apexian Pharmaceuticals (Inst), Birdie Pharmaceuticals (Inst), AbbVie (Inst), Constellation Pharmaceuticals (Inst), Syndax (Inst) Travel, Accommodations, Expenses: Asana BioSciences

\section{A. Dimitrios Colevas}

Stock and Other Ownership Interests: Gilead Sciences, Pharmacyclics Consulting or Advisory Role: COTA, KeyQuest Health, Loxo Oncology, Atara Biotherapeutics, Aduro Biotech, Cue Biopharma, Pfizer

Research Funding: Bristol-Myers Squibb, AstraZeneca, Innate Pharma, CellSight Technologies, Tessa Therapeutics

\section{Timothy O'Rourke}

Research Funding: Asana BioSciences (Inst)

\section{Sujata Narayanan}

Employment: Genentech

Stock and Other Ownership Interests: Roche

Kyriakos Papadopoulos

Consulting or Advisory Role: Bayer AG (Inst), ArQule (Inst)

Research Funding: AbbVie (Inst), Medlmmune (Inst), Daiichi Sankyo (Inst), Regeneron Pharmaceuticals (Inst), Sanofi (Inst), ARMO BioSciences (Inst), ArQule (Inst), Calithera Biosciences (Inst), Curegenix (Inst), Incyte (Inst)Merck (Inst), Peloton Therapeutics (Inst), ADC Therapeutics (Inst), 3D Medicines (Inst), Formation Biologics (Inst), EMD Serono (Inst), Syros Pharmaceuticals (Inst), Mersana (Inst), OncoMed (Inst), MabSpace Biosciences (Inst), Jounce Therapeutics (Inst)
George A. Fisher

Stock and Other Ownership Interests: Seattle Genetics (I)

Honoraria: Merck, Genentech, Ipsen, Novartis

Consulting or Advisory Role: Ipsen, Genentech, Roche, Merck, Jounce Therapeutics

Research Funding: Newlink Genetics (Inst), XBiotech (Inst), Genentech (Inst), Roche (Inst), EpicentRx (Inst), Merck (Inst), Forty Seven (Inst), Aduro Biotech (Inst), Novartis (Inst)

Travel, Accommodations, Expenses: Merck Sharp \& Dohme

Victor Villalobos

Consulting or Advisory Role: Janssen Pharmaceuticals, Eli Lilly, Novartis, AbbVie, Ignyta, Agios, Epizyme

Travel, Accommodations, Expenses: Eli Lilly, Janssen Pharmaceuticals, Xencor, GenMab, Apizyme

Susan S. Prohaska

Employment: Forty Seven

Stock and Other Ownership Interests: Forty Seven, Amgen

Consulting or Advisory Role: Forty Seven

Patents, Royalties, Other Intellectual Property: Inventor on patent developed at Stanford and assigned to Forty Seven

Other Relationship: Stanford University

Maureen Howard

Stock and Other Ownership Interests: Forty Seven

Consulting or Advisory Role: Cartherics

Patents, Royalties, Other Intellectual Property: Methods for Achieving

Therapeutically Effective Doses of Anti-CD47 Agents, Stanford University, Nov 2014, WO 2014/1494772; Methods for Achieving Therapeutically Effective

Doses of Anti-CD47 Agents, Stanford University, Feb 2014, US 502667359

Muralidhar Beeram

Honoraria: Genentech

Consulting or Advisory Role: Novartis (I)

Speakers' Bureau: Genentech

Research Funding: Eli Lilly (Inst), Zymeworks (Inst), Mersana Therapeutics (Inst)

Mark P. Chao

Employment: Forty Seven

Leadership: Forty Seven

Stock and Other Ownership Interests: Forty Seven, Hepatx

Honoraria: D2G Oncology

Consulting or Advisory Role: Chimera Bioengineering

Patents, Royalties, Other Intellectual Property: Inventor on several patents from Stanford University licensed to Forty Seven, inventor on patents generated from Forty Seven

Travel, Accommodations, Expenses: Forty Seven

Balaji Agoram

Employment: Forty Seven

Stock and Other Ownership Interests: Forty Seven

James Y. Chen

Employment: Forty Seven

Stock and Other Ownership Interests: Forty Seven

Consulting or Advisory Role: Forty Seven

Research Funding: Forty Seven

Travel, Accommodations, Expenses: Forty Seven

Jie Huang

Employment: Forty Seven

Travel, Accommodations, Expenses: Forty Seven

Matthew Axt

Employment: Forty Seven

Employment: Kaiser Permanente (I)

Stock and Other Ownership Interests: Forty Seven 
Jie Liu

Stock and Other Ownership Interests: Forty Seven

Patents, Royalties, Other Intellectual Property: Stanford/Forty Seven: CD47, SIRPa, CD99

Jens-Peter Volkmer

Employment: Forty Seven

Stock and Other Ownership Interests: Forty Seven

Patents, Royalties, Other Intellectual Property: Co-inventor on technologies that have been licensed to Forty Seven

\section{Ravindra Majeti}

Employment: Roche (I), Genentech (I)

Leadership: Forty Seven

Stock and Other Ownership Interests: Forty Seven, Roche (I), Genentech (I), Amgen (I), Imago BioSciences

Honoraria: Roche, Genentech, Pharmacyclics

Consulting or Advisory Role: Forty Seven, Biomarin

Research Funding: Celgene, Bluestar Genomics

Patents, Royalties, Other Intellectual Property: Numerous patents related to CD47 targeting and therapeutics that have been licensed to Forty Seven

Irving L. Weissman

Employment: Forty Seven

Leadership: Forty Seven

Stock and Other Ownership Interests: Forty Seven

Consulting or Advisory Role: Forty Seven

Patents, Royalties, Other Intellectual Property: Many patents licensed by

Stanford to Forty Seven, patents licensed to Systemix that were sold to Sandoz and Novartis, inventions for Systemix, patent pending in the brain stem cell field as part of Stem Cells cofounded in 1997 and sold in 2016

Travel, Accommodations, Expenses: Forty Seven

Other Relationship: Stem Cells (I)
Chris H. Takimoto

Employment: Forty Seven

Leadership: Forty Seven

Stock and Other Ownership Interests: Johnson \& Johnson, Forty Seven

Patents, Royalties, Other Intellectual Property: Patent proposals filed related to using anti-CD47 antibodies as cancer therapy at Forty Seven

Travel, Accommodations, Expenses: Forty Seven

Dana Supan

Research Funding: Forty Seven (Inst)

Heather A. Wakelee

Honoraria: Novartis, AstraZeneca

Research Funding: Genentech (Inst), Roche (Inst), Pfizer (Inst), Eli Lilly (Inst), Celgene (Inst), AstraZeneca (Inst), Medlmmune (Inst), Exelixis (Inst), Novartis (Inst), Clovis Oncology (Inst), Xcovery (Inst), Bristol-Myers Squibb (Inst), Gilead Sciences (Inst), Pharmacyclics (Inst), ACEA Biosciences (Inst)

Travel, Accommodations, Expenses: AstraZeneca

Rhonda Aoki

Research Funding: Forty Seven (Inst), Celldex Therapeutics (Inst)

Mark D. Pegram

Employment: Loxo Oncology (I)

Consulting or Advisory Role: Genentech, Pfizer, Novartis, Amgen, AstraZeneca, Merck

Travel, Accommodations, Expenses: Genentech, Pfizer, Novartis, DAVA

Pharmaceuticals

Sukhmani K. Padda

Consulting or Advisory Role: Janssen Pharmaceuticals, G1 Therapeutics, AstraZeneca, AbbVie

Research Funding: EpicentRx (Inst), Forty Seven (Inst), Bayer AG (Inst) 


\section{APPENDIX}

\section{Patient Eligibility}

Additional patient eligibility criteria included having malignancy assessable for response by Response Evaluation Criteria in Solid Tumors (RECIST) for solid tumors or International Working Group criteria for lymphomas; ECOG performance status 0-2; life expectancy at least 3 months, and negative urine or serum pregnancy tests for all women of child-bearing potential.Exclusion criteria included primary brain tumors, patients with brain metastases, red blood cells (RBCs) transfusion dependence, hemoglobin $<9.5 \mathrm{~g} / \mathrm{dL}$, neutrophils $<1.0 \times$ 109/mL, AST/ALT > 5 times upper limits of normal (ULN), Bilirubin $>$ 2.5 times ULN, serum creatinine $>1.5$ times ULN, and significant medical conditions that would substantially increase the medical risks of participation in the study.

\section{Methods}

Dose-limiting toxicity exceptions: Grade 3 anemia; Grade 3 nausea, vomiting, diarrhea, or infusion reactions in the absence of premed ication (including influenza-like symptoms, myalgias, fever, chills, headache, or acute pain) that resolves to $\leq$ Grade 2 within $\leq 72$ hours after medical management (eg, supportive care) has been initiated; Grade 3 fatigue that resolves to $\leq$ Grade 2 within 2 weeks on study Grade 3 hyperbilirubinemia, isolated electrolyte abnormalities, or alanine aminotransferase and/or aspartate aminotransferase elevations that resolve to $\leq$ Grade 2 with supportive care within 72 hours and is not associated with other clinically significant consequences; Grade 3 tumor lysis syndrome or related electrolyte disturbances (hyperkalemia, hypophosphatemia, hyperuricemia) that resolve to $\leq$ Grade 2 within 7 days; Grade 3 or 4 lymphopenia.

\section{Routine Laboratory Assessments}

Laboratory assessments included routine blood counts with reticulocyte counts, general chemistry panels, serum uric acid, phosphorous, haptoglobin, D-Dimer, thrombin time, plasma fibrinogen, serum folic acid, prothrombin time, activated partial thromboplastin time, total iron and transferrin saturation, and urinalysis. All patients had a blood type and screen and direct antigen test performed at screening. Electrocardiograms were obtained at baseline and days 8 , 29 and 57 of therapy. Peripheral blood smears were evaluated for blood cell morphology pre- and post-5F9 dosing on days 1 and 8 and on days $2,4,8,11,15,22$ and 29 . High resolution retinal photographs were performed at screening, and on days $1,2,4,8,9,11$ and 15 to assess for retinal microangiopathy; however, retinal imaging was halted in Part B due to a lack of correlative findings.

\section{Pharmacokinetics and Anti-Drug Antibodies}

Blood samples for pharmacokinetics (PK) monitoring were collected on day 1 pre-dose, and at the end of infusion and on day 8 at pre-dose and 0,1 , and 2 hours after the end of infusion. Additional PK samples were collected on days 9 and 30 , and pre- and post-dose on days 11 (if dosed), 15, 22, 29, 36, 43, 50, and 57 and finally at the end of study treatment. Samples were shipped to a central laboratory for analysis of 5F9 concentration in serum using a validated ELISA Assay. ${ }^{15}$ The assay was specific to 5F9 and had a lower limit of quantification of approximately $200 \mathrm{ng} / \mathrm{mL}$. Noncompartmental analysis (NCA) was performed using NCA for R software package to estimate PK parameters such as terminal half-life and area-under-the-concentration (AUC) curve. ${ }^{19}$

Anti-drug antibodies (ADA) were collected pre-dose and then every 4 weeks through week 17 , and then on weeks $25,33,49$ and 30 days after the last dose of 5F9. Samples were shipped to a central laboratory for ADA analysis. ${ }^{15}$ The ADA analysis followed a three-tiered approach, consisting of screening, confirmatory, and titer steps with pre-specified false positive rates of $5 \%$ and $1 \%$ for the screening and confirmatory steps, respectively. The cut points were generated using in-study samples.

\section{Pharmacodynamics and Tumor Biopsies}

Blood samples for CD47 receptor occupancy assessments on RBC and WBC were collected on day 1 and 8 pre- and post-dosing with 5F9 and on days 15, 22, 29, 36, 57 and end of study treatment. Receptor occupancy measurements were quantitated by using a flow cytometry assay with a tagged fluorescent anti-CD47 antibody to assess saturation of CD47 binding sites on RBC and WBC obtained from patients.

In the mandatory tumor biopsy cohort, patients were required to undergo mandatory tumor biopsies that were obtained pre-treatment and during week 6 of treatment. Tissue specimens were formalin- fixed and paraffin-embedded and subjected to immunohistochemical stains using an anti-lgG4 antibody. Because the levels of endogenous IgG4 levels are low in most tissues, treatment related changes could detect the presence of $5 \mathrm{F9}$ in tissues during treatment.

TABLE A1. Summary of Geometric Mean (Geometric \%CV) $C_{\max }$ and AUC Values after the First (Priming), Second, and Fifth Doses at All Dose Levels (Study SCI-CD47-001; Data Supplement)

Week 1

Week 2

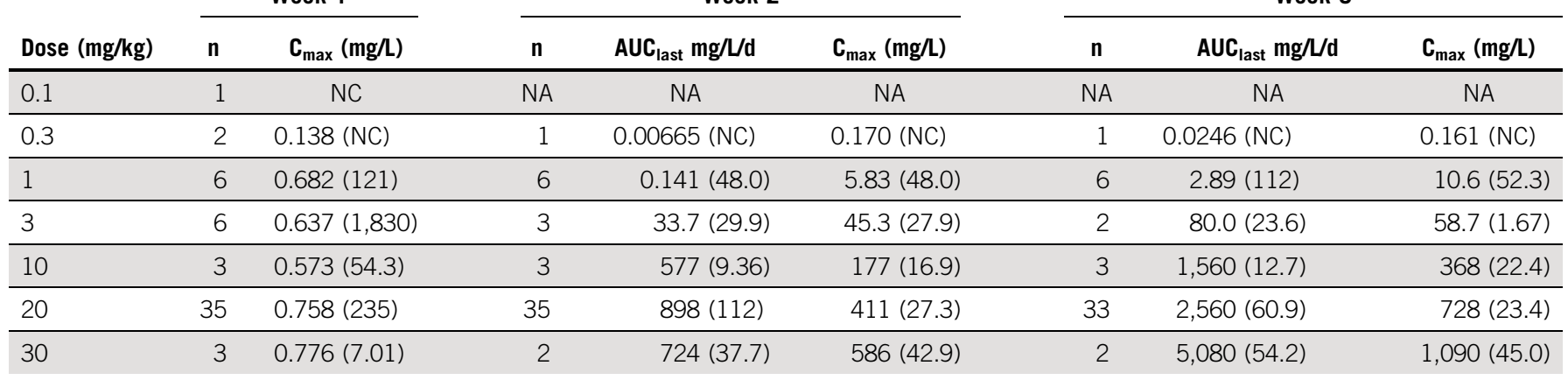

Abbreviations: AUC, area-under-the-concentration; NA, not applicable; NC, not calculated 